\title{
Modeling Distributed Energy Resource Dynamics on the Transmission System
}

\author{
Ross T. Guttromson, Member, IEEE
}

\begin{abstract}
As distributed energy resource (DER) projects grow in popularity, there has been little focus on their potential to influence the dynamic stability of a transmission system. If implemented in large enough numbers, DER equipment may feasibly be leveraged to provide ancillary services such as spinning reserve, system inertia, or system stabilization. To determine the potential impacts of DER on future transmission grid stability, dynamic models of DER load combinations were created, reduced in order, and scattered throughout Central and Southern California within an existing Western Systems Coordinating Council (WSCC) dynamic model. Evaluation of the composite WSCC-DER dynamic model allows correlations to be drawn between various DER properties and the transmission grid stability. Key findings show that increasing DER inertia tends to destabilize the transmission system. The cause of this counterintuitive relationship requires further study but likely is related to the high impedance separating the DER from the transmission grid.
\end{abstract}

Index Terms-Distributed energy resources, distributed generation, dynamic performance, small-signal stability, transient stability.

\section{INTRODUCTION}

D YNAMIC MODELING of distributed energy resources (DERs) provides a unique challenge because most DER devices are power electronics based, and manufacturers do not readily provide model data, if any exist at all. The analysis presented in this paper does not attempt to identify transmission system response to DER but rather to show how specific properties of DER change the stability of the transmission system.

For the given problem, identifying the precise dynamic response of a real DER device becomes less important. The DER model response simply can be assumed and implemented into a larger Western System Coordinating Council (WSCC) model, and the overall composite system then can be analyzed. When one property of DER (such as inertia) is changed, a new composite model is evaluated, and changes to the WSCC-DER system response become meaningful.

The cases discussed in this paper have initially modeled DERpenetrated distribution systems with round-rotor synchronous generators attached to IEEE type-ST1 static exciters using a

Manuscript received February 22, 2002; revised May 6, 2002. This work was supported by the Assistant Secretary of Energy Efficiency and Renewable Energy, Office of Power Technologies, U.S. Department of Energy, through the Consortium for Electric Reliability Technology Solutions (CERTS). The Pacific Northwest National Laboratory is operated by Battelle for the U.S. Department of Energy under Contract DE-AC06-76RL01830.

The author is with the Pacific Northwest National Laboratory, Richland, WA 99352, USA.

Digital Object Identifier 10.1109/TPWRS.2002.804957

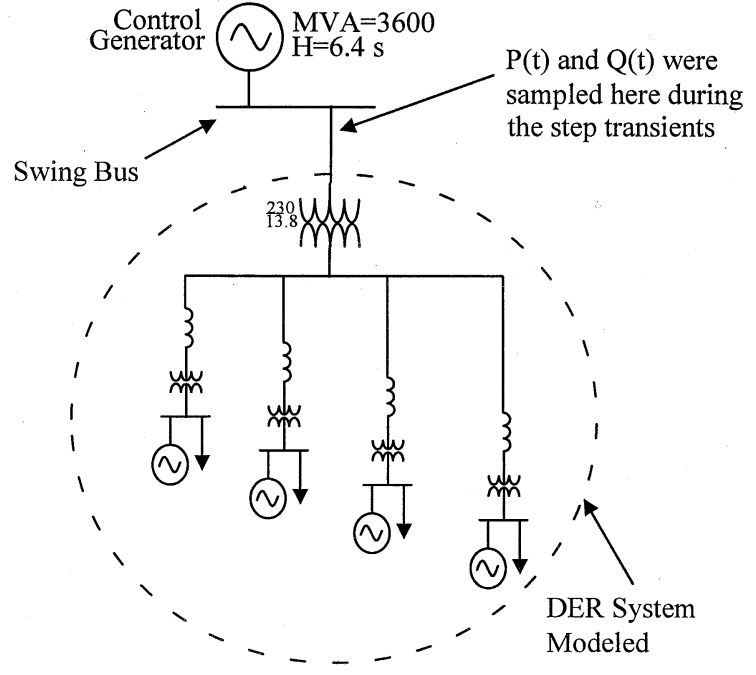

Fig. 1. System for obtaining model signals.

General Electric (GE) positive sequence load flow/positive sequence dynamic simulation (PSLF/PSDS) transient simulation package [1] (see Fig. 1). Use of these models supports the convenient manipulation of properties such as inertia constants, exciter gain values, and various other control parameters. Although properties such as inertia and exciter gain do not apply to power electronic DER devices, their concepts do apply because DER controllers can be designed to react in many different ways to voltage and frequency disturbances.

After the DER-penetrated distribution system models were created, they were reduced in order using Prony analysis and then converted to transfer functions; thus, the transfer function (reduced-order model) exhibited the same dynamic response as the original DER-penetrated distribution system model shown in Fig. 1. Copies of the reduced-order model were scaled and attached to a large 10000 bus WSCC model for further dynamic analysis using GE's PSLF/PSDS software. The reduced-order models represent the dynamics of DER-load combinations and do not change the steady-state load-flow conditions of the grid. Therefore, each DER reduced-order model influences the grid in the same manner as several matched DER sources and loads would, modulating real and reactive power $(P$ and $Q)$ in response to voltage and frequency changes on the grid.

Sensitivity studies were performed by modifying one parameter at a time within the DER dynamic models shown in Fig. 1 (such as inertia or exciter gain) and then recreating new reduced-order models described by (2). The same 10 000-bus 
WSCC model was used with the new reduced-order model to determine the influence on the WSCC transmission system.

\section{DER IMPACTS ON SYSTEM STABILITY}

Two different approaches were employed to find the impacts on WSCC dynamic stability from the DER. Both methods are based on DER system data as described in Appendix A.

The first method was based on analyzing the dynamic modes of a composite WSCC-DER system. A sensitivity study was conducted that involved changing the inertia constants of the DER and then analyzing the composite WSCC-DER ringdown response to a 0.5-s 1400-MW Chief Jo Brake event. Six key signals in California were captured from each dynamic simulation result. Prony analysis was then used to identify the common modes of these signals between 0 and $1 \mathrm{~Hz}$. The analysis of these signals quickly revealed that each mode's residue had a greater impact on stability than did its decay factor. Therefore, residues for each signal were used as the benchmark for stability.

A second method was based on observing changes to existing stability limits resulting from changes to DER properties. One of these stability limits restricts the imported power flow into Southern California. This Southern California Import Transmission (SCIT) nomogram requires a minimum amount of local system inertia to safely import power. As the amount of locally dispatched generation (hence inertia) increases, Southern California power imports may safely be increased as well. Violation of the limit results in a partial WSCC system breakup after a worst-case $N-1$ casualty. Therefore, when a DER property was changed, the new SCIT limit for the composite WSCC-DER system was identified and used as a benchmark for system stability. An increase in the SCIT limit indicates a benefit to system stability, whereas a decrease in the SCIT limit indicates a detriment to system stability.

\section{MODEL REDUCTION}

Exhibiting the dynamic influences of DER on the transmission grid requires the use of a very large dynamic model. Since DER models were to be proliferated throughout an already massive WSCC dynamic model, it was necessary to reduce the model order of each DER system to enable computational analysis.

The Prony method was selected to reduce the model order. The numerical advantages of Prony analysis make it well suited for approximating higher order signals with an optimum loworder model. Applications of the Prony method to power systems are shown in [2]-[4].

Consider a general continuous signal $y(t)$ that is to be modeled by

$$
\hat{y}(t)=\sum_{i=1}^{n} B_{i} e^{\lambda_{i} t}
$$

where $B_{i} \in C$ is the output residue for the continuous-time pole $\lambda_{i} \in C$, and $\lambda_{i} \neq \lambda_{j}$ is the output residue for $j \neq i$. The Prony method identifies the residues, poles, and $n$ that force (1) to be the least-squares fit to $y(t)$.
Using GE's PSLF/PSDS transient simulation package [1], a dynamic model of a DER-penetrated distribution system was created as described in the Appendix. This system was connected to a control generator, representing an infinite bus as shown in Fig. 1. Real and reactive power oscillations between the distribution system and the control generator were captured and converted into a state-space model using the Prony method. A significant reduction in the model order was achieved because many of the poles embedded in the signals were composed of frequencies not relevant to power system dynamic interactions and, thus, were discarded.

The first controlled transient was a clean step in voltage at the point of connection of the distribution system. The step change in voltage was made by changing the base voltage of the swing bus, which was attached to the control generator residing outside of the distribution system, while holding frequency of the control generator constant. Since the control generator was attached to the swing bus, it did not see the step change to the swing bus base voltage and, therefore, did not react to the voltage step transient. During the voltage step, the frequency was held constant by making the inertia of the control generator much greater than the total inertia contained within the distribution system. Therefore, the distribution system frequency was allowed to swing against the control generator but was essentially unable to influence its rotor speed.

The resulting real power signal $P v(t)$ had its initial conditions removed and was used to extract a linear model $G p v(s)$, which is the relationship of real power transfer with respect to a change in voltage. The reactive power-to-voltage model $G q v(s)$ was made in the same manner.

The second controlled transient was a step in frequency of the control generator while voltage was held constant. The step change was made by raising the mechanical power of the control generator during one time step of the dynamic simulation. Thus, a precise change in frequency was implemented on the control generator in the span of a one-quarter cycle. This transient produced signals $P f(t)$ and $Q f(t)$, which were used to create linear models $G p f(s)$ and $G q f(s)$.

Inertia was shown to be high enough to keep frequency relatively stable during each transient. This was verified by capturing the frequency signal during the voltage step transient, which varied less than $10^{-5}$ per unit (pu) and entering it into the frequency models $G p f(s)$ and $G q f(s)$. The results showed that $P$ and $Q$ were influenced by the undesired frequency transient on the order of $10^{-4} \mathrm{pu}$.

Prony analysis was performed on each signal. Poles and residues were selected based on their relative energy, damping, and frequency content so that the sampled signal was reproduced to a tenth-order system or less. After a transfer function for each signal was created, the input signal $U(s)$ was removed from it by removing the visible pole near zero. The resulting model is described by

$$
\left[\begin{array}{c}
\Delta P \\
\Delta Q
\end{array}\right]=\left[\begin{array}{ll}
\frac{G p v(s)}{\Delta v} & \frac{G p f(s)}{\Delta f} \\
\frac{G q v(s)}{\Delta v} & \frac{G q f(s)}{\Delta f}
\end{array}\right] \cdot\left[\begin{array}{c}
\Delta v \\
\Delta f
\end{array}\right]
$$

Making the reduced-order model with zero initial real or reactive power transfers ensures that it would influence only the 


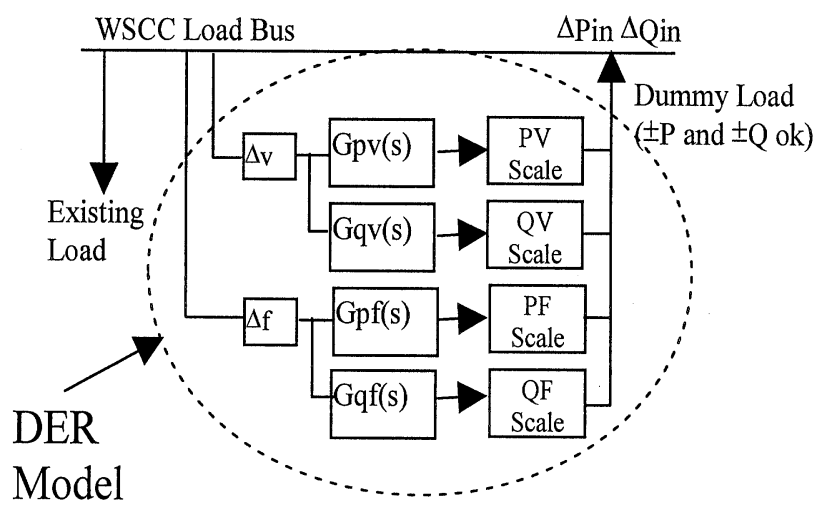

Fig. 2. Implementation of the DER model.

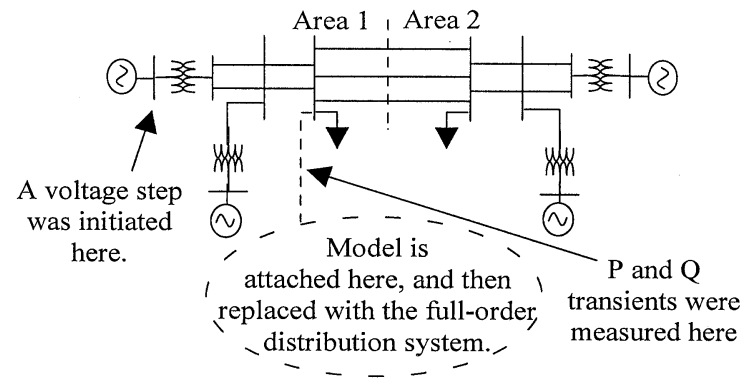

Fig. 3. Two-area test system used to validate model response.

dynamic response. In reality, the magnitude of the dynamic response will be larger or smaller, depending on the size of the distribution system. For this reason, a scaling factor is multiplied to the output of the reduced-order model, scaling the dynamic response of the model with respect to the size of the DER model that it represents. Details concerning the scaling factor are discussed in Section V.

Next, the model was converted into state-space form and programmed into a custom PSDS dynamic model using GE's EPCL programming language [1]. It was implemented to create the composite dynamic model, as shown in Fig. 2.

\section{Model VALidation}

The next step was to validate the model. A test system that would represent the dynamics of a large power system, yet be as small as possible, was desired. For that reason, the test system was modeled using a four-generator, ten-bus power system, similar to that used in [6]. The 3600-MVA test system supported two-area power system dynamics and included dynamic models for each steam turbine, round rotor generator, static exciter, and one power system stabilizer (see Fig. 3).

After the real and reactive power transients between the two connected systems were captured, the full-order distribution system model was removed from the four-generator test system and replaced with the reduced-order model (see Fig. 3). The same disturbance was initiated, and again, $P$ and $Q$ transients were measured between the four-generator system and the reduced-order model. The results are shown in Fig. 4. As can be seen, the model appears to do a very good job at simulating the dynamic response of the original distribution system.
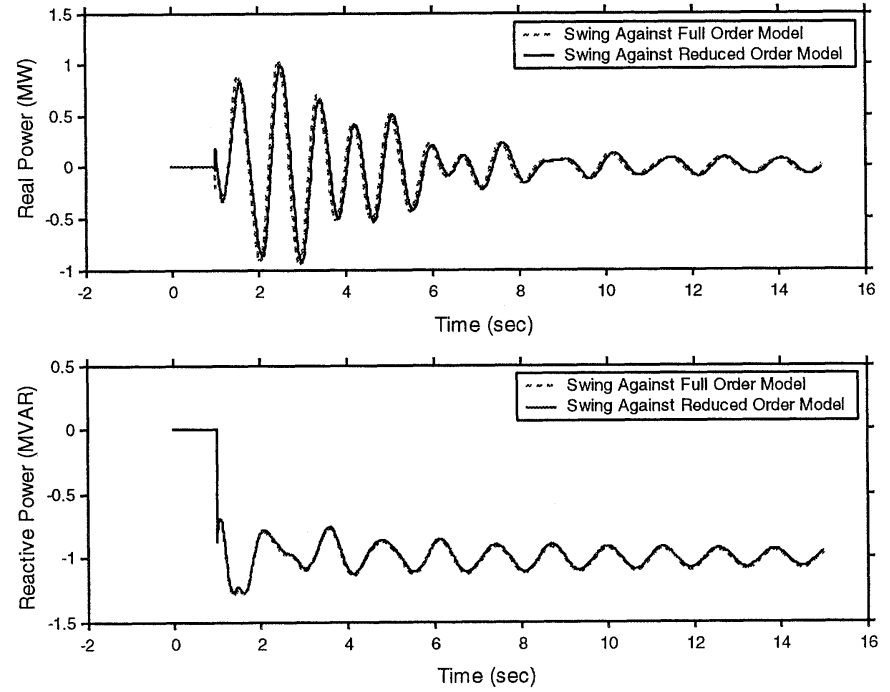

Fig. 4. Comparisons of actual and modeled response for a voltage step transient.

\section{Siting AND SCALING THE DER Models}

Deciding where to site each DER model within the WSCC model involved several considerations. One would expect DER equipment to be dispersed deep within the distribution systems. However, WSCC load-flow cases do not model distribution lines and model very little subtransmission infrastructure. Siting decisions are complicated further by the fact that the existing WSCC case contains more than 10000 buses, making it extremely difficult to add distribution and subtransmission lines to the WSCC model. Therefore, loads within WSCC power-flow cases were aggregated at the transmission level, and the DER models were sited at existing WSCC load buses.

It was also decided that the size of each DER model should be in proportion to the size of the existing loads at each load bus. This does not imply that a DER model exhibits the dynamics of existing loads but, rather, that the aggregate rating of DERs will be in proportion to the amount of existing load.

For consistency in evaluation, it was essential that the same DER model be implemented throughout the WSCC but not at all the same rating. For this reason, it was necessary to scale the model output to account for larger or smaller DER installations. One could apply linear scaling so that models representing $200 \mathrm{MW}$ of DER and load simply provided a response that was ten times that of a 20-MW model. However, it was found that a linearly scaled response was so exaggerated that the WSCC composite model solution diverged shortly after a minor disturbance was initiated. Therefore, a nonlinear scaling factor was introduced, which scaled the dynamics of extremely large loads to a realistic value. Scaling factors were obtained by modeling a subtransmission system and placing it between a DER-penetrated distribution system and a control generator. Controlled voltage and frequency-step transients were initiated, and the magnitudes of the resulting $P$ and $Q$ transients were recorded. This process was repeated several times with different values of load and generation assumed in the DER-penetrated distribution system until several data points were obtained. Curves fits 

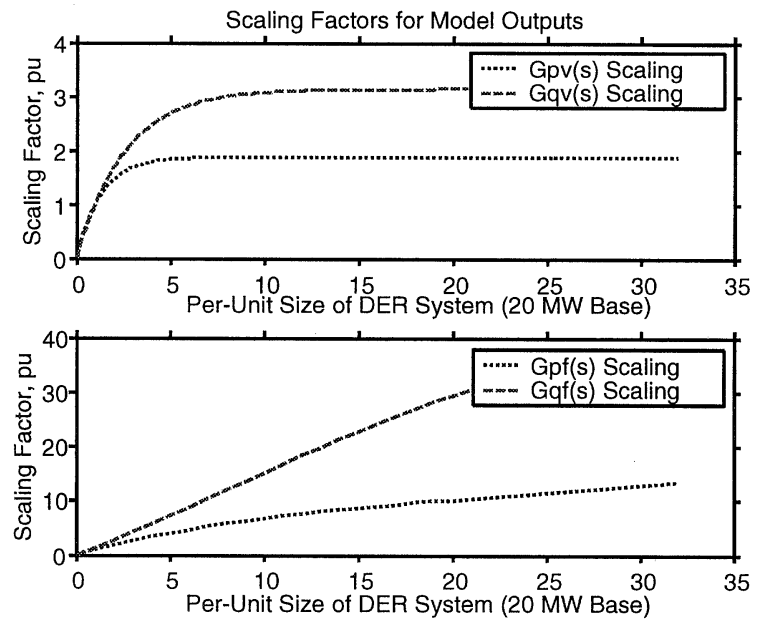

Fig. 5. Curves used to scale DER and load size.

of the data points are shown in Fig. 5 and were used to scale each reduced-order model within the WSCC composite model.

\section{MODEL APPROXIMATIONS}

The major approximations to the model, which are known, have been listed here.

\section{A. System Nonlinearity}

Issues such as magnetic saturation, discrete-tap changers, load shedding, and over and undervoltage tripping are examples of nonlinearities that cannot be modeled with a linear modeling technique, such as the Prony method.

- Nonlinearities with respect to model size were approximated. DER models were scaled to exhibit the characteristics of larger or smaller installations.

- Nonlinearities with respect to input were ignored. The models were created from the response to a specific step change in frequency and voltage. The linear nature of the model dictates that if the input is twice as large, the response will also be twice as large. However, this assumption is clearly not true in power systems.

\section{B. Direct Voltage Control}

In a distribution system penetrated by DER equipment, the system's feedback to the transmission grid provides more than just a real and reactive power response. An actual system may directly influence voltage as well as real and reactive power. This is especially evident in cases of voltage instability, where increasing the reactive power injection contributes to a voltage collapse [7]. Although the injection of real and reactive power into the transmission grid causes an indirect influence on system voltage, the reduced-order model does not provide a voltage output, and hence, the response of the model never will exactly match that of the system.

\section{Dynamics of the Control Generator}

The reduced-order model was designed to represent the DERpenetrated distribution system only and not that of the control

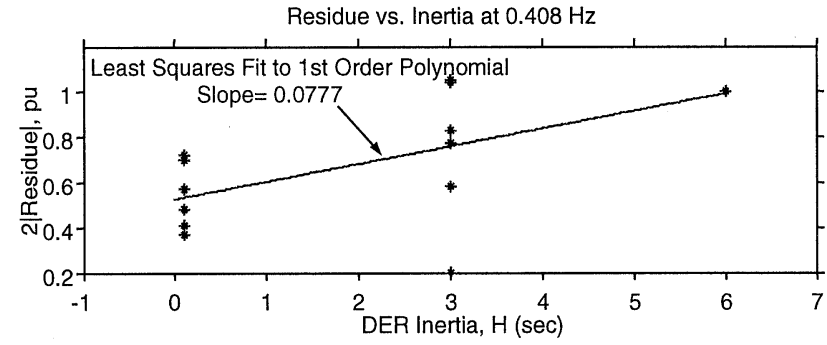

Fig. 6. WSCC-DER system stability as a function of DER inertia at $0.406 \mathrm{~Hz}$.

TABLE I

IMPACTS OF DER INERTIA ON WSCC SYSTEM STABILITY By MEASUREMENT OF SYSTEM MODES

\begin{tabular}{l|c}
\hline \multicolumn{2}{c}{ Key WSCC Modes } \\
\multicolumn{2}{c}{ During a 1400-MW Chief Jo Brake Ringdown } \\
\hline Mode, Hz & $\frac{d}{d H} \mid 2 \cdot$ residue $\left.\right|_{p u}$ \\
\hline 0.311 & -0.0028 \\
\hline 0.408 & +0.078 \\
\hline 0.455 & -0.0091 \\
\hline 0.633 & -0.11 \\
\hline 0.729 & -0.11 \\
\hline
\end{tabular}

generator. However, the model inherently includes some characteristics of the control generator. These effects are undesirable but, unfortunately, will always be present in this type of model.

\section{Simulation RESUlts}

As described in Section II, two different approaches were employed to find the impacts of DER properties on WSCC dynamic stability. The first method was based on analyzing the dynamic modes of the composite WSCC-DER system, with results shown in Table I and Fig. 6.

The data points shown in Fig. 6 were obtained by analyzing six key California power signals noted in the Appendix. The residues of each signal were per-unitized, grouped into modes, and plotted against their corresponding value of DER inertia. The process was repeated for all other significant modes between 0 and $1 \mathrm{~Hz}$, with results shown in Table I.

The second method was based on observing changes to SCIT stability limits, resulting from changes to DER properties such as inertia, exciter gain, or load type. Fig. 7 explains the concept further.

The SCIT stability limits can be expanded or contracted by changing the amount of inertia in Southern California (i.e., the amount of dispatched generation). As the amount of inertia in Southern California increases, the SCIT nomogram expands, permitting more power imports into Southern California. When models of DER are placed in the Southern California region, an " $X$ " denotes how DER models affect the SCIT nomogram limits, exhibiting benefits or detriments to the system stability. The results of the SCIT analyzes are shown in Fig. 7 and Tables II-IV. 


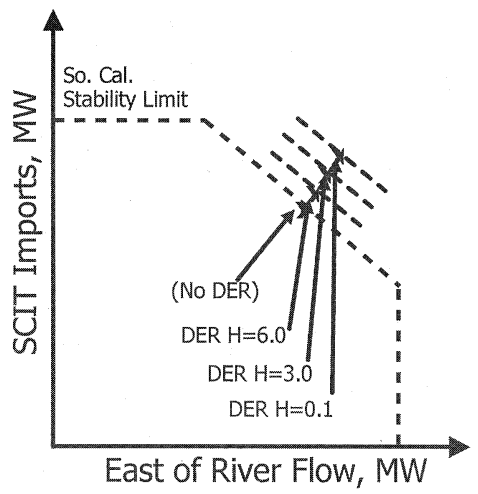

Fig. 7. Changes to Southern California Import Transmission (SCIT) limits as DER models are added.

TABLE II

IMPACTS OF DER INERTIA ON WSCC SYSTEM STABILITY BY CHANGES TO SCIT LIMITS

DER - Inertia Sensitivity Studies from SCIT

$(\mathrm{Ka}=200$; loads as in Appendix I)

\begin{tabular}{l|c}
\hline DER Inertia H, sec & $\begin{array}{c}\text { Change in SCIT } \\
\text { Imports, MW }\end{array}$ \\
\hline $\mathrm{H}=6.0$ & +318 \\
\hline $\mathrm{H}=3.0$ & +637 \\
\hline $\mathrm{H}=0.1$ & +702 \\
\hline
\end{tabular}

TABLE III

IMPACTS OF DER EXCITER RESPONSE ON WSCC SYSTEM STABILITY BY CHANGES TO SCIT LIMITS

\begin{tabular}{|c|c|}
\hline \multicolumn{2}{|c|}{$\begin{array}{c}\text { DER - Exciter Gain Sensitivity Studies } \\
\text { from SCIT } \\
(H=6 ; \text { loads as in Appendix I })\end{array}$} \\
\hline $\begin{array}{l}\text { Exciter Gain KA, } \\
\text { p.u. }\end{array}$ & $\begin{array}{l}\text { Change in SCIT } \\
\text { Imports, MW }\end{array}$ \\
\hline $\mathrm{Ka}=200$ & +318 \\
\hline $\mathrm{Ka}=20$ & +567 \\
\hline $\mathrm{Ka}=5$ & +454 \\
\hline Q Regulation & +417 \\
\hline
\end{tabular}

TABLE IV

IMPACTS OF DER LOAD TYPE ON WSCC SYSTEM STABILITY BY CHANGES TO SCIT LIMITS

DER - Load Type Sensitivity Studies from SCIT $(\mathrm{DER}=$ Constant $\mathrm{P}+\mathrm{jQ})$

\begin{tabular}{l|c}
\multicolumn{1}{c|}{ DER Load Type } & $\begin{array}{c}\text { Change in SCIT } \\
\text { Imports, MW }\end{array}$ \\
\hline $\begin{array}{l}100 \% \text { Induction Motor for P } \\
\text { and Q }\end{array}$ & +174 \\
\hline $\mathrm{P}=100 \% \mathrm{IP}$ & +787 \\
$\mathrm{Q}=100 \% \mathrm{~B}$ & \\
\hline $\begin{array}{l}\mathrm{P}=31 \% \mathrm{P}(\mathrm{f})+32 \% \mathrm{IP}(\mathrm{f})+ \\
\mathrm{Q}=100 \% \mathrm{G}(\mathrm{f})\end{array}$ & +634 \\
$\begin{array}{l}\text { Same as Southern } \\
\text { California) }\end{array}$ & \\
\hline $\begin{array}{l}\text { Standard Load Profile (as } \\
\text { shown in Appendix I) }\end{array}$ & +582 \\
\hline
\end{tabular}

\section{CONCLUSION AND FURTHER STUDY}

\section{A. Effects of DER Inertia}

As can be seen from Table I, increasing the DER inertia benefits the transmission system stability for this WSCC load-flow case at the $0.311-, 0.455-, 0.633-$, and $0.727-\mathrm{Hz}$ modes but is a detriment at the $0.408-\mathrm{Hz}$ mode.

Results shown in Table II confirm this counterintuitive conclusion that DER inertia may not be providing any benefit to the WSCC system stability.

A new WSCC case was obtained from the California Independent System Operator (ISO), with power-flow conditions substantially different from those of the previous cases. For this new case, no DER models were attached, and an attempt was made to observe the system instability resulting from operation outside the SCIT nomogram. Since rotational inertia is a physical property of each generating unit, the only way to change the inertia within an area is to change the dispatch (i.e., put generation units online or take them offline). However, doing this changes more than the inertia. It also changes the voltage and reactive power characteristics at the bus where the generating unit was connected. Therefore, to verify the relationship of grid stability versus Southern California inertia (as presented in the SCIT nomogram), the generator dispatch was not changed in Southern California. Instead, the inertia constants $(H)$ in the generator models in the Southern California area were scaled. The results of this exercise showed that system stability could be maintained when Southern California inertia was reduced by an amazing $80 \%$ below the minimum limits shown on the SCIT nomogram. This implies that the SCIT nomogram is much less a function of Southern California inertia and more of a function of the Southern California generation base dispatched.

Since small changes in inertia were shown to have little influence on the system's small-signal stability, it is not overly surprising that DER inertia might not benefit the system stability. However, why it would hurt the system stability is not obvious. One possibility is that since the DER units were placed behind so much reactance, their response to a system disturbance was dramatically phase shifted, causing a destabilizing effect. If this were the case, it would also appear feasible that a controller could be designed to compensate for this phase lag and provide a net benefit to system stability. Perusing this idea is a topic of future study.

\section{B. Effects of DER Voltage Control}

It is well understood that high-gain exciters often introduce negative damping, causing small-signal instability of the power system [5], [7]. Therefore, they are often implemented with power system stabilizers (PSSs) to compensate for this instability. Although the WSCC model includes many PSSs, not one was retuned to account for the high-gain excitation within the DER models.

The results of this sensitivity study exhibited the predictable phenomena. Increasing DER exciter gain aided the stability of the system up to a high value of $K a$, after which, increasing values of $K a$ were detrimental to system stability, attributed by its introduction of negative damping. The addition of a constant 
reactive-power regulator ( $Q$ regulator) to the DER exciter had an effect similar to that of lowering the exciter feedforward gain.

These results imply that DERs can have a beneficial impact on transmission stability by supplying reactive power at the distribution level.

\section{Effects of DER Load Type}

For the load-type sensitivity studies, the DERs themselves were made without any dynamics. However, the load dynamics were preserved. Instead of using round-rotor synchronous generator models with static exciters, the DER sources were modeled as constant $P$ and $Q$ injection. When a system disturbance occurs, system voltage typically decreases as a result of the disturbance. Since the DER sources inject constant $P$ and $Q$ while the loads within a distribution system are voltage dependent, there will be a net $P$ and $Q$ export from the distribution system during most disturbances. This net $P$ and $Q$ injection appears to enhance the system's stability, as can be seen from Table IV.

\section{APPENDIX \\ WSCC-DER SYSTEM DATA}

DER models used in the SCIT analysis were constructed as shown in the following with impedances obtained from [8]:

- We have one 25-MVA, 230/13.8-kV transformer.

- There are four 600-A (605-kcmil) distribution feeders with lengths of $0.5,1,1.5$, and $2 \mathrm{mi}$.

- There are four 6-MVA, 13.8/0.480-kV XFMRs (one per feeder).

- All DERs and loads were connected at the end of their respective feeders at $480 \mathrm{~V}$;

- Each feeder modeled one 5-MVA synchronous generator with an IEEE-type ST1 exciter (no governor). Hence, there was one DER per feeder, totaling four DERs within the model.

- Loads were dynamic, sited adjacent to DERs, and characterized by

a) $8 \%$ constant power;

b) $42 \%$ constant impedance;

c) $30 \%$ induction motored;

d) $20 \%$ frequency dependent.

DER models were sited within the WSCC dynamic model as follows:

- 224 models into Southern California (areas 22, 24, and 26), totaling $9.5 \mathrm{GW}$.
The following WSCC signals were used for the Chief Jo Brake ringdown model analysis:

1) Telsla Station voltage magnitude;

2) Lugo Station voltage magnitude;

3) Malin-to-Round Mtn real-power transfer;

4) Malin-to-Round Mtn reactive-power transfer;

5) Devers-to-Palo Verde real-power transfer;

6) Midway-to-Vincent real-power transfer.

\section{ACKNOWLEDGMENT}

The author extends special thanks to P. Mackin, formerly of the California ISO, L. Szot, and L. Tobias of the California ISO, and colleagues Dr. J. Hauer and J. Dagle of the Pacific Northwest National Laboratory.

\section{REFERENCES}

[1] User Manual: Positive Sequence Load Flow (PSLF), Positive Sequence Dynamic Simulation (PSDS) and Engineers Program Control Language $(E P C L)$, General Electric Co., Schenectady, NY.

[2] D. J. Trudnowski, "Order reduction of large-scale linear oscillatory system models," IEEE Trans. Power Syst., vol. 9, pp. 451-458, Feb. 1994.

[3] J. F. Hauer, C. J. Demeure, and L. L. Scharf, "Initial results in Prony analysis of power system response signals," IEEE Trans. Power Syst., vol. 5, pp. 80-89, Feb. 1990.

[4] J. M. Johnson and D. J. Trudnowski, Users Manual: DSITools Ringdown Analysis Tool. Columbus, OH: Battelle Memorial Inst., 1998, vol. 2.

[5] M. K. Donnelly, J. E. Dagle, D. J. Trudnowski, and G. J. Rogers, "Impacts of the distributed utility on transmission system stability," IEEE Trans. Power Syst., vol. 11, pp. 741-746, May 1996.

[6] M. Klein, G. L. Rogers, and P. Kundur, "A fundamental study of inter-area oscillations," IEEE Trans. Power Syst., vol. 6, pp. 914-921, Aug. 1991.

[7] P. Kundur, Power System Stability and Control. New York: McGrawHill, 1994.

[8] J. D. Glover and M. Sarma, Power System Analysis and Design. Boston, MA: PWS-Kent, 1987.

[9] C.-Y. Wu, 1998 California Operating Studies Subcommittee (OSS) Handbook. Folsom, CA: California Independent System Operator, 1998.

Ross T. Guttromson (M'01) received the B.S.E.E. degree from Washington State University, Pullman, in 1995.

Currently, he is a Research Engineer with the Power Systems Energy Technology Division, Pacific Northwest National Laboratory, Richland, WA. He is also enrolled in the graduate program at Washington State University.

He was with R. W. Beck Engineering and Consulting, Seattle, WA, from 1999 to 2001 and with the Generator Engineering Design Group, Siemens-Westinghouse Power Corporation, Orlando, FL, from 1995 to 1999. 